\title{
Dante e Montale: la voce, l'allegoria, la trascendenza
}

\author{
Pierpaolo Antonello
}

In qualsiasi storia letteraria italiana i nomi di Dante e Montale compaiono, temporalmente polarizzati, come i cardini di una tensione e di una tradizione poetiche che forse non hanno eguali nella cultura Europea. Non c'è letteratura che sia stata così marcata da un lato da un poeta fondatore tanto esuberante e costrittivo, e dall'altro da una tradizione che, forse anche per questa sua origine, è rimasta sostanzialmente lirica e ha dato il meglio di sé sotto il segno di questa costellazione. ${ }^{1}$ Ed è forse per questo che la voce del fiorentino continua ancora a risuonare intertestualmente nei poeti di ogni generazione, e ancor di più nel Novecento e in particolare nel suo poeta che maggiormente lo rappresenta, Eugenio Montale, per i motivi che cercheremo di illustrare in questo nostro contributo, soprattutto raccogliendo le voci critiche che meglio hanno saputo illuminare il rapporto in questione.

Per sua stessa natura e definizione la parola poetica è una parola che si auto-fonda e si auto-costituisce nell'atto stesso del suo prodursi e rinnovarsi. Una parola che pretende e impone di essere riconquistata ad ogni atto di scrittura. Soprattutto quella parola poetica contemporanea (in tutte le possibili nuove scuole o avanguardie) che vuole programmaticamente porsi sempre come forza che oltrepassa la tradizione e la rinnova e che non può avere e credere poeta più moderno di Dante. A ragione, infatti, Contini distingue fra la linea 'sperimentale' (dantesca) delle nostre lettere e la linea 'formale' (o petrarchesca), quasi a sottolineare che nella tradizione italiana non c'è poeta che più di lui abbia fatto i conti con l'innovazione di un linguaggio e di una lingua, con la creazione quasi ex-nihilo della propria lingua. Non c'è poi poeta tradizionalmente più engagé dal punto di vista politico, animato da "una totale spregiudicatezza verso il reale" (Contini 363) e da una costante sovversione nel pensiero e nei canoni, pur rimanendo nel quadro di una struttura cosmologica e filosofica tradizionale.

Sulla contemporaneità di Dante e sulla sua ennesima riscoperta e studio, Montale rileva nel suo famoso intervento del 1965 (dove Maria Corti si rammarica sia fallita la convergenza di racconto di Eliot e Montale) (Corti 55), che "se Dante è patrimonio universale [...] la sua voce oggi possa giungere a tutti come mai forse avvenne in altri tempi e come forse non sarà più possibile 
in futuro" (Sulla 15). Della stessa idea è Giuseppe Prezzolini, ripreso da Ierardo, che ci aiuta a dire della modernità dantesca: "Dante può dirsi una scoperta del nostro tempo, benché le sue idee non corrisposero allo sviluppo dell'Italia. Soltanto l'artista universale, il moralista intransigente attirano il lettore moderno, meravigliato che ci sia stato al mondo un carattere così portante" (Ierardo 9). Forse proprio perché una figura di poeta tanto radicale e forte era quello che la poesia del Novecento - e probabilmente tutta la cultura italiana - stava inseguendo che Dante è ritornato a risuonare nella voce di poeti anche molto diversi fra loro come D'Annunzio e Ungaretti, Govoni e Pasolini, Cardarelli e Montale.

\section{Dante e Montale}

L'influenza di Dante su Montale, ovvero il ricorso a Dante da parte di Montale, non sembra mai essere stata posta in dubbio dalla critica. Il materiale e i loci paralleli sono così sovrabbondanti e il riconoscimento in questo senso da parte di Montale così esplicito da non poter mettere in conto una seria refutazione della consistenza di questo schema. Gli interventi critici, numerosi ormai a riguardo, testimoniano ampiamente in questa direzione, ${ }^{2}$ facendo di Montale, come suggerisce Mario Petrucciani, "lo scrittore per cui più di ogni altro, forse, fra i massimi del secolo [...] si è discusso di dantismo" (164). Eppure è lo stesso Montale che si interroga su questa influenza, sul significato della presenza del fiorentino nella sua lingua e nella sua poesia: "che cosa significa l'opera di Dante per un poeta oggi? Esiste un suo insegnamento, un'eredità che non possiamo raccogliere?" (Sulla 32). E questa influenza, come vedremo, è un oscillare tra distanza irrecuperabile e richiamo continuo, quasi necessario di un poeta che è stato il poeta di intere generazioni a livello mondiale e che diventa ora in maniera rinnovata, più complessa, il nuovo Virgilio, maestro di mestiere, di raffinato lavoro poetico, più che guida sincreticamente artistica e spirituale di elevazione. E se Giorgio Orelli giunge a dire che talvolta Montale gareggia con Dante ("L'upupa" 250) - come Dante a suo tempo ricordava che il suo concedere plauso ai maestri Guido o Virgilio, comportava nello stesso momento l'assunzione che lui li aveva, quasi per incarico divino, superati - vorremmo ricordare come Montale stesso abbia tentato di sposare piuttosto la lettura del Singleton di un Dante "miracolato", scriba di Dio e per tanto modello inavvicinabile, stella polare impossibile da raggiungere e sfidare, perché di fatto unico poeta esistito: "di fronte a Dante non esistono poeti" insiste Montale (Sulla 33). ${ }^{3}$

Questa appropriazione di Dante da parte di Montale non si limita - e anzi va ben oltre - al richiamo intertestuale vagamente allusivo o una "virtualità esplosiva di un frammento che possa inserirsi a far spicco in un contesto novecentesco" come suggerisce Ramat (311). In questo senso vale la pena 
notare qualche presenza così rumorosamente sonora e evidente che ricorda anche a chi non ne fosse convinto come i fili fra Montale e Dante siano saldi. Basti vedere, ad esempio, come da Par. 5.133-35:

\section{Sì come il sol che si cela essi stessi per troppa luce, come 'l caldo ha rose le temperanze di vapori spessi ${ }^{4}$}

la frase dantesca migri, sempre in incipit di verso, in Gloria del disteso pomeriggio, portandosi appresso tutte le connotazioni di questa immagine di vapori dissolti e di arsura:

e più e più si mostrano d'attorno per troppa luce, le parvenze, falbe.

Il sole, in alto, - e un secco greto.

E ancora il famoso passo del ramarro del nono mottetto:

Il ramarro, se scocca sotto la grande fersa delle stoppie

che richiama Dante in Inf. 25.79-80:

Come 'I ramarro solto la gran fersa dei dí canicular, cangiando sepe

dove l'immagine, così palesemente montaliana - il meriggiare, la canicola estiva, l'immobilità delle cose nell'estate mediterranea - sembra derivarsi proprio da Dante, quasi che il poeta ligure ci tenesse a dire che anche quanto è più suo e più lo distingue, rientra in un debito, in un prestito dal poeta sommo; quasi che quanto è venuto dopo Dante fosse solo una chiosa al grande poema sacro.

\section{La voce}

Silvio Ramat in un suo saggio spiega che a differenza di Eliot, approdato a Dante per lettura ideologica, Montale vi indugia "portato da un orecchio musicale ed una facoltà intuitiva" (316). ${ }^{6} \mathrm{E}$ in questa nostra analisi comparata, vorremmo proprio partire dal dato che ha condotto da principio Montale a Dante, un dato in qualche modo più "superficiale", ma allo stesso tempo più squisitamente poetico, nel senso jakobsiano, della sostanza poetica, ovvero fonetica e "letterale" della lingua; ciò che distingue il linguaggio poetico 
dagli altri linguaggi rispetto alla sua funzione: quella voce, quella musica che risuona sino alle sue note più riposte: "lettere, non ho che lettere" recita Montale nella penultima lirica di Mediterraneo. È un approccio che sembra quasi scontato parlando di studi poetici, di parole che "cantano" la lingua; eppure, come suggerisce Orelli, la critica italiana non ha saputo prestare la dovuta "attenzione alla Lautsprache, al suono della voce poetica, in particolare agli "armonici", come dice Montale in una lettera a Cambon (91), a quei valori sonori e timbrici (...) che concorrono all'assestamento del cosiddetto ritmo semiotico" (Orelli, Accertamenti 18). Ovviamente non si tratta di ricorrere a fonosimbolismi di sorta, piuttosto a "parole atte per le loro 'risorse' foniche a iscriversi in un 'insieme' solidale quanto basta, di cui potenziano il contenuto" (19). Eppure nonostante questa presunta disattenzione critica invocata da Orelli, ${ }^{7}$ niente ci sembra più naturale che sottolineare una relazione di assonanze e di richiami timbrici e eufonici nel tessuto allitterativo montaliano importati dalla lettura delle rime dantesche. "Dante ha una voce tutta sua fin dagli inizi" tende a precisare lo stesso Montale quasi a dirci cosa conta veramente nella struttura poetica (Sulla 29). La proverbiale scabrezza della lingua del poeta ligure, la sua durezza, il suo tratteggiare un paesaggio essenziale e secco, non pud che trovare come primo e assoluto maestro Dante, "dove ritorna il giuoco delle rime aspre e difficili" (Sulla 30), in particolare il Dante più cupo e scabro, ma in questo senso più modemamente efficace, dell'Inferno, dove il paesaggio risuona nella sua durezza e cattiveria di una impossibilità di speranza, di redenzione e di salvezza, che come vedremo più avanti costituira anche un nucleo tematico privilegiato dell'opera montaliana. Pipa anche senza spingere a fondo la descrizione in merito, lo rivela come primo avvicinamento alla relazione Montale-Dante, richiamando una corrispondenza di rime sin dai più alti momenti di Ossi di seppia (16). In particolare in Meriggiare pallido e assorto, dove la rima "sterpi/serpi" riprende chiaramente l'episodio della selva dei suicidi in Inf. 13.37-39:

Uomini fummo, e or siam fatti sterpi:

ben dovrebb'esser la tua man pì̀ pia, se state fossimo anime di serpi.

Dove il riferimento dantesco viene evidenziato anche dalla scelta del vocabolario: in entrambi i casi i cespugli d'arbusti vengono definiti come pruni (vedi Inf. 13.32). Ancora sotto la rima "formiche/biche" ci rimanda alla decima bolgia di Inf. 29.64-66:

si ristoran di seme di formiche;

ch'era a veder per quella oscura valle

languir li spirti per diverse biche. ${ }^{8}$ 
Un altro luogo famoso del primissimo Montale, il "rivo strozzato che gorgoglia" di Spesso il male di vivere, si dispiega attraverso un ennesimo richiamo "infernale" (Inf. 7.125):

quest'inno si gorgoglian nella strozza.

E come non rilevare in queste occasioni quello che Orelli suggeriva poc'anzi: una solidarietà forte fra l'intonazione della voce e il contenuto dei versi in questo modo potenziati. Il richiamo al paesaggio infernale è quindi doppio: verbale e tematico, nel dispiegamento retrospettivo, per l'appunto, di un racconto di cammino ("Spesso il male di vivere ho incontrato") e di un paesaggio di desolazione e morte privo di ogni speranza ("il rivo strozzato che gorgoglia", "il cavallo stramazzato"). Dove invocando la anti-manzoniana "divina Indifferenza" il poeta si chiama fuori dalla tradizione etico-religiosa che ha istituito lo sfondo di pensiero di un poeta quale Dante. Nel primo mottetto la parola dantesca appare poi con una lapidarietà esemplare priva di scampo: "E l'inferno è certo".

Lo sai: debbo riperderti e non posso.

Come un tiro aggiustato mi sommuove ogni opera, ogni grido e anche lo spiro salino che straripa dai moli e fa l'oscura primavera di Sottoripa.

Paese di ferrame e alberature a selva nella polvere del vespro. Un ronzio lungo viene dall'aperto, strazia come unghia ai vetri. Cerco il segno smarrito, il pegno solo ch'ebbi in grazia da te.

E l'inferno è certo.

Qui i richiami danteschi sono evidenti e dispiegati a più livelli. Il tema è l'allontanamento, il riperdere, lo smarrire come smarrirsi, dove il venir meno di una persona cara (una luce, una Beatrice?) trasforma di schianto il paesaggio della costa ligure, sul molo di Genova, in una selva d'alberi e di gru, quasi a oscurare l'orizzonte. Ripa è poi toponimo dantesco per eccellenza che si porta appresso l'asperità dei significanti distinguibili in straripa, oscura, vetri, strazia nonché il richiamo alle rime in Inf. 9.1-3:

In su l'estremità d'un'alta ripa che facevan gran pietre rotte in cerchio, venimmo sopra più crudele slipa; 
dove ovviamente non viene ripreso il solo parallelismo delle rime ma l'intero impianto sonoro della terzina: "estremita", "gran pietre rotte" "sopra...stipa". Orelli - lettore che più ha in orecchio le dinamiche di queste assonanze - a questo proposito si spinge oltre, e rintraccia una parentela timbrica anche in quello "spiro salino che straripa" e che richiama la famosa immagine del Paradiso, una immagine comunque non pacificata, né salvifica ma che riferisce a un Dante terreno, a un destino di esilio e di sofferenza immerso in assonanze "sapide": "Tu proverai SÌ come SA di SALE / lo pAnE ALtrui, e come è duro cALLE / lo SCendere e 'L SALIR per L'ALtrui ScALE”' (Orelli, Accer-tamenti 24).

\section{L'allegoria}

È sempre il Ramat che ci aiuta a introdurre un susseguirsi di temi presunti nei testi montaliani dicendoci che Montale "eे penetrato in Dante per grandi e suggestionanti bagliori, senza entrare nell'ordine e negli schemi della sua piena razionalità" (319). D'altronde è impossibile per un autore del Novecento sposare la costruzione mistico-razionalista di un uomo del tardo medioevo. E Montale si rende conto della difficoltà di giungere a una piena comprensione del modello, a sviscerare fino in fondo e a capire adeguatamente, con la nostra Gestalt concettuale ristrutturata dall'Umanesimo, il mondo poetico-allegorico di una mente medievale come quella di Dante. Quando Montale afferma "Dante non pud essere ripetuto" e "resta estraneo ai nostri tempi" (Sulla 33), costruisce una sorta di resistenza concettuale, di distanza, ma allo stesso tempo una presa di coscienza delle difficolta di innestare un ritmo narrativo e un apparato ideologico e morale così lontani e dissimili da quelli della contemporaneità, nel proprio racconto. La tensione è del resto estremamente produttiva.

Per alcuni critici sembra che questa distanza-vicinanza di voci si innesti sul meccanismo di descrizione poetica che appare più lontano dalla nostra poetica moderna (nonostante il recupero baudeleriano): l'allegoria. A proposito è necessario premettere che la chiave allegorica di avvicinamento alla relazione Dante-Montale non è chiara ed è stata momento di dibattito critico. In particolare Barański punta il dito contro quelle letture, Pipa e Jacomuzzi in testa, che hanno deciso di sposare questa ipotesi critica, e fa perno sul silenzio esegetico di lettori attenti come Contini, Corti, Mengaldo e Cambon, dove, come nel caso di Ramat e Orelli, non si neghi decisamente ogni possibilità di lettura in questa direzione (Barański, "Dante" 13).

Leggendo da vicino il dibattito, ci si accorge che, ad esempio, Jacomuzzi, in un suo intervento in proposito, dopo aver sostenuto che "l'esaltazione del discorso analogico-metaforico, è il punto d'attacco di Montale con il discorso dantesco, il luogo del suo innesto organico che spiega e dà senso alla estrema 
varietà e ricchezza dei riscontri testuali già accertati dalla lettura critica" (219), non provvede a descrivere in concreto esempi in cui il discorso montaliano si venga ad articolare in senso allegorico. Pipa, che sposa la lettura di Jacomuzzi, d'altro canto fallisce nel ricostruire una lettura troppo libera e forzatamente allegorica di Fuscello teso dal muro che, a nostro avviso misinterpreta il testo montaliano (19-24).

Non è nostro interesse ora controbattere punto per punto la lettura di Pipa. Possiamo però suggerire alcune critiche possibili e dire che, ad esempio, l'interpretazione della nave come una nave pirata ci sembra oltre modo "fantasiosa", soprattutto perché Pipa si basa sull'uso da parte di Montale di termini quali "trealberi", "ciurma", "tolda" che all'orecchio di Pipa suonano come desueti, mentre per un ligure, nato sul mare a inizio secolo, costituivano patrimonio linguistico quotidiano e rispondevano a una nominazione nomenclatoria precisa e fedele. "Tolda" inoltre pud essere stato preferito a "coperta" per l'assonanza allitterativa con i successivi "timone" e "traccia". Un altro appunto che potremmo avanzare è che il procedimento allegorico, come Dante insegna, prevede che non si indichi esplicitamente il referente a cui la trasfigurazione allegorica viene a riferirsi. Nel caso scelto da Pipa, Montale scrive:

Fuscello teso dal muro

sì come l'indice d'una meridiana che scande la carriera del sole e la mia, breve.

Qui si ha chiara l'indicazione che si parla del poeta e della sua vita (come anche Pipa asserisce) e per tanto il procedimento di interpretazione puo avvenire per via analogica e non certo allegorica.

Prendendo le mosse da questo dibattito, ci verrebbe da dire che lo slancio allegorico è affidato alla fiducia nella capacità di rimandare a un livello altro, a un regno di significati che appaiono con la coerenza di una fede. Montale puo avere questa tensione, questo movimento e moto spirituale, che si fonda sulla "certezza di un senso" (Camon 83), ma non giunge mai a compimento, non giunge mai a questo significato, per impossibilità ontologica o per mancanza di forza o di fede ("e questo tu potrai, chissà, non io"). ${ }^{9}$

L'interesse e l'attenzione di Montale per Dante, da come la possiamo intendere noi con i nostri pochi strumenti concettuali, si situa piuttosto su quel ritorno alle cose, all'oggetto istantaneamente inventato dalla lingua dantesca. Montale in modo esplicito afferma: "Io parto sempre dal vero, non so inventare nulla [...]. Il dato realistico, però, è sempre presente, è sempre vero". E ancora in questa direzione su Dante: "Dante crea gli oggetti nominandoli e le sue sintesi sono fulminee [...]. Dal pertugio del sensibile, dall'esaltazione delle forme Dante evade cosi dalle strette del pensiero scolastico" (Sulla 30). Non bisogna poi dimenticare come in quel saggio, Dante ieri e oggi, Montale 
punti la sua attenzione sul Dante storico, ricostruendo il profondo legame fra biografia e fare letterario. Un modo per avvicinarlo a sé, a un suo sentire di poeta che lavora con la materia calda dell'esistenza per "transumanarla". E in fondo questa estrema dedizione alle cose e alle descrizioni icasticamente precise, limpide, mirate che fa di Dante un poeta assolutamente moderno - e qui lo rincorre Montale. Diremo quindi con il Ramat che il correlativo "oggettivo' di eliotiana memoria (il "correlativo obiettivo" come lo traduce Montale stesso) venga a invocarsi in questa circostanza più che una qualsiasi dimenticata e lontana forma allegorica. Del resto e lo stesso autore di Ossi di seppia che ci ricorda che "il nostro mondo non conosce più visioni ma il mondo di Dante è ancora quello di un visionario" (Sulla 30). ${ }^{10}$

\section{La trascendenza}

\section{Scrive ancora Ramat:}

"Il dramma dei moderni è semmai nella constatata impossibilità a vincere l'empiria, e quella realtà che nella poesia contemporanea si è indicata come "metafisica", può essere giudicata tale solo all' interno del suo universo empirico, e corrisponde allo sforzo della parola a raggiungere una rappresentatività che trascenda il movente fenomenico che l'ha suscitata e nutrita in partenza". (323)

In Montale, attorniato da una realtà oggettuale statica e forte, dispiegata in natura e persone e eventi che portano infisso il segno de "il male di vivere", si inserisce indubbiamente un forte desiderio di trascendenza, di individuare "l'anello che non tiene", la "via di fuga", e di andare, come canta in Maestrale, "più in la". La religiosità laica del poeta ligure, se di religiosità si pud parlare, e tutta trattenuta in una morale scabra, interiormente sofferta. Si spinge fino ad un passo dalla rivelazione che non viene: "Il viaggio finisce qui", e non riuscendo a conquistarla per sé, la spera per le persone che gli stanno vicino, come splendidamente scrive in Casa sul mare, una poesia che rimanda con potenza seduttiva al poeta che ha cantato il "più in la":

Tu chiedi se così tutto vanisce in questa poca nebbia di memorie; se nell'ora che torpe o nel sospiro del frangente si compie ogni destino. Vorrei dirti di no, che ti s'appressa l'ora che passerai di là dal tempo; forse solo chi vuole s'infinita, e questo tu potrai, chissà, non io. Penso che per i più non sia salvezza, ma taluno sovverla ogni disegno, passi il varco, qual volle si ritrovi. 
Ecco che il recupero di Dante segue questo passo. Si tratta di ascoltare un poeta che fisicamente, intellettualmente e spiritualmente ha raccontato il "più in la", un poeta che " $s$ "infinita" sua trascendenza a cui l'uomo moderno non ha accesso, che la percepisce forse possibile ma che non ha la forza di sperare. Il cammino di Montale non giunge a conversione ma si ferma alle soglie del mistero del tempo e della sua capacità di consunzione.
Il viaggio finisce qui
Il cammino finisce a queste prode
che rode la marea con moto alterno.

Non c'è la temporalità ciclica e perfetta delle sfere empiree, ma il susseguirsi ininterrotto del flusso di un tempo che rode, sbriciola, non lascia scampo, implacabile.

Al fianco una sorda incomunicabilità con chi poeta o altro:

Il tuo cuore vicino che non m'ode salpa già forse per l'eterno.

Il dramma temporale, dell'inesorabile castrazione del tempo nei confronti della memoria, con squisita ascendenza dantesca, ritorna in un altro famosissimo luogo montaliano: Non recidere, forbice, quel volto che proviene da $P a r$. 26.9: "lo tempo va dintorno con la force". ${ }^{13}$

Nel dispiegarsi di questo movimento verso l'altrove, verso il più in là, c'è chi ha individuato per Montale (il Montale dei Mottetti, soprattutto) una figura analoga a quella di Beatrice nel contesto dantesco, lume, ragione, guida e angelo salvifico. In Incontro si legge:

La tua vita è ancora tua: tra $\mathrm{i}$ guizzi rari

dal giomo sparsa già. Prega per me

allora ch'io discenda altro cammino

che una via di città.

Una indicazione di poetica esplicita è lo stesso Montale a fornirla, attraverso un richiamo di discorsi paralleli chiari ed esplicativi. In Intenzioni Montale racconta di se stesso e di donne cantate nelle sue opere e dice: "Ho proiettato la Selvaggia o la Maledetta o la Delia (...) dei Mottetti sullo sfondo di una guerra cosmica e terrestre, senza scopo e senza ragione; e mi sono affidato a lei, donna o nube, angelo o procellaria" (568). In un altro testo invece torna su Dante ripetendo: "Ma chi è costei? Certo, in origine, donna reale; ma qui e altrove, anzi dovunque, visiting angel, poco o punto materiale" (Sulla 91). 
Le sue donne acquistano così questa particolare valenza di double bind, elemento sacro che ha una valenza ad un tempo salvifica e negativa, "angelo e procellaria", e che rimanda, più che al Dante della Commedia dove Beatrice e ormai radicalmente trasfigurata, alla Vita Nova e alla donna Petra e alla Donna gentile le quali, sempre secondo Montale, "avrebbero dovuto essere inventate di sana pianta se non fossero mai esistite: perché non si può immaginare un processo di salvezza senza la controparte dell'errore e del peccato" (Sulla 21). Ecco che il parallelismo si rafforza e insieme diventa asimmetrico: attraverso un previo errore e peccato si giunge alla salvezza. Ma Montale prescrive, come detto, salvezza al solo angelo, mai a se stesso. Non abbandonerà mai la spiaggia del purgatorio (o dell'inferno) ${ }^{14}$ rimanendo a contemplare di 1i, malinconicamente, l'incapacità di seguire la sua Beatrice che si "infinita": "e questo tu potrai, chissà, non io".

E inoltre nel "mottetto" già citato:

Cerco il segno

smarrito, il pegno solo ch'ebbi in grazia

da te.

E l'inferno è certo.

E ancora in Stanze dove ricorre la stessa dinamica di una salvezza che è lasciata ad altri:

Tocchi il segno, travalichi. Oh il ronzio dell'arco ch'è scoccato, il solco che ara il flutto e si richiude! Ed ora sale l'ultima bolla in su. La dannazione è forse questa vaneggiante amara oscurità che scende su chi resta.

Concede Montale, comunque e sempre, l'insidia del "miracolo", i miracoli che sono sempre possibili e sempre in agguato alla nostra porta e quindi sposa la tesi del Pietrobono dicendo che "la donna miracolosa non solo visse ma fu un effettivo miracolo" a indicare, come sostiene Maria Corti, "la condizione estrema di trascendenza prodotta dall'oggetto poetico (oggetto talismano definisce splendidamente Contini)" (59) e che ci riporta ancora alle parole di Montale che ripete con una convinzione che sembra squisitamente dantesca: "il miracolo era per me evidente come la necessita. Immanenza e trascendenza non sono separabili" (Sulla 565). Sotto questo segno, pensiamo, si risolve la distanza e insieme la vicinanza di Dante e Montale: attraverso un assunzione ideologica comune, che Dante riesce a colmare completamente per indole, storia, epoca, forza poetica e religiosa, e che Montale può solo sperare e vedere apparire come "miracolo", un miracolo che nella biografia montaliana 
forse accade proprio esclusivamente nella poesia del solo poeta totalizzante che, sempre secondo Montale, la nostra tradizione abbia dato: Dante appunto.

\section{Stanford University}

NOTE

1 Eppure questa comune e ormai trita considerazione riguardo le sorti e i destini della nostra storia letteraria sembra così sapientemente ri-spiegata da Montale stesso, quando asserisce che la prosa italiana ha fallito in parte non per il peso eccessivo di una tradizione poetica troppo imperante, ma per l'esatto contrario, perché non è riuscita a capire la prosa insita in Dante e la sua forza: "si ha l'impressione che molti episodi della Commedia siano stati scritti di getto con pochi pentimenti, realizzando così quella grande prosa nascosta dentro le maglie del ritmo e delle rime che se fosse stata compresa e portata a compimento ci avrebbe risparmiato secoli di prosa curiale, togata" (24). Altri riferimenti a questo proposito si vedano anche alle pp. 102 e 173 dello stesso testo.

2 Oltre ai testi a cui si farà riferimento esplicito, è importante ricordare i contributi di Cambon e Comello. Un ponte critico, una meditazione notevole che ha agito nella lettura montaliana di Dante è quella di lma Brandeis (studiosa che nel contesto critico anglo-americano ha saputo raccontare con sapienza entrambi i poeti). A questo proposito si leggano i commenti stessi di Montale alle pagine della Brandeis (31-32).

3 Si legga anche a p. 592.

4 Tutti i corsivi usati nelle citazioni sono miei.

5 "Con lui nasce e forse muore la poesia italiana" (Montale, Sulla 601).

6 Come si è visto in esordio, Montale stesso parla esplicitamente di "voce" nel richiamarsi a Dante (Sulla 15).

7 Del resto possiamo vedere come Barański spieghi esplicitamente che "for Montale Dante's Inferno, and perhaps also his canzoni petrose, seem to have constituted a storehouse aiding his own poetic search for harsh sound effects and suitable vocabulary to reflect a negative vision of life" (“A note" 399).

8 "Per la rima scricchi-picchi si pensa alle rime Osterlicchi-Tambernicchi-scricchi in Inf. 32.26, 28,30 , che i critici classicisti disapprovarono severamente per la stranezza dei suoni e per la singolarità stessa delle immagini che comportavano" (Bonora $31 \mathrm{n}$ ).

9 Chi anche sposa, come Luperini, l'ipotesi di una costitutiva forma allegorica nella poesia montaliana, muovendo proprio da questo assunto, lascia inevasa un'analisi testuale estesa e approfondita, accontentandosi di riferimenti troppo scoperti, come ovvia L'allegoria, o approdando alla luckasiania "allegoria vuota" caratteristica dell 'ultimo Montale (279-325).

10 Più avanti nella stessa pagina Montale si dimostra poco interessato alla lettura allegorica che Eliot fa di Dante, richiamandosi invece ad altre analisi più interessanti, ancora a testimoniare che il pemo della ricezione montaliana di Dante non verte sull'elemento allegorico: "Messa da parte la sicurezza di chi ritiene di poter spiegare l'allegoria in ogni parte, restano più interessanti gli interpreti che, vedendo nella Commedia un immensa ragnatela di corrispondenze, cercano di ricondurre ogni filo, o meglio qualche filo, al suo centro".

$11 \mathrm{Al}$ di là di un vago richiamo leopardiano, Bonora spiega come infinitarsi sia "una di quelle invenzioni per le quali Montale sembra si sia educato alla scuola di Dante" (127-28n).

12 Per il termine "varco" si possono fare vari riferimenti, come ad esempio Inf. 12.26 "E quello accorto gridò: 'Corri al varco"”; Pur. 19.43, "Venite, qui si varca!"; Pur. 23.28 "la bella donna che mi trasse al varco"; in questo caso quello che toma immediatamente alla memoria è $P$ ar. 27.82-84, "si ch 'io vedea di là da Gade il varco / folle di Ulisse". 11 richiamo ad Ulisse ritorna infatti nella poesia successiva a Casa sul mare, I morti, tematicamente dantesca come poche altre. 
13 Ma è la poesia successiva a Casa sul mare che vieta ogni speranza per il poeta. I morti - e non poteva essere altrimenti - risuona continuamente di sonorità e tensioni dantesche: "la ferrigna costa", "il gorgo sterile", "il groppo torbido", "il ribollio", "larve" e soprattutto quel finale che richiama, come detto, il "varco" d'Ulisse. Se Dante riesce a passare il varco e a salvarsi, Montale rincorre la figura di Ulisse, viaggiatore senza guida e senza fede, ormai parte di quei morti che sono "larve rimorse dai ricordi umani", "fiati / senza materia o voce / traditi dalla tenebra; / ed i mozzi / loro voli ci sfiorano pur ora / da noi divisi appena e nel crivello / del mare ci sommergono...". E in questi versi finali risuona, chiaro ed esplicito, il richiamo al "folle volo" di Inf. 26.125 e al suo epilogo "infin che'l mar fu sovra noi richiuso" (Inf. 26.142).

14 Potremo richiamare questa metafora della costa e della spiaggia che è presente come momento di limite e di indugio in Dante e che viene ripresa in maniera continua da Montale. Oltre alla più scontata e "fisica" spiaggia del Purgatorio, in apertura dell' Inferno ritroviamo "uscito fuor del pelago alla riva" (1.23); "ripresi via per la piaggia diserta" (1.29).

\section{OPERE CITATE}

Alighieri, Dante. La Divina Commedia. Ed. Giuseppe Villaroel. Milano: Mondadori, 1985.

Barański, Zygmunt. "A Note on Montale's Presumed Dantism in Meriggiare pallido e assorto". Italica 56.4 (1979): 394-402.

. "Dante e Montale: The Threads of Influence". Dante comparisons. Ed. Eric Haywood and Barry Jones. Dublin: Irish Academic Press, 1985. 11-48.

Bonora, Ettore. La poesia di Montale. Ossi di seppia. Padova: Liviana, 1982.

Brandeis, Ima. The Ladder of Vision. London: Chatto \& Windus, 1960.

Cambon, Glauco. "Montale dantesco e bruegelliano". Aut Aut 35 (1956): 37 1-91.

Camon, Ferdinando. Il mestiere di poeta. Milano: Garzanti, 1965.

Comello, Toni. "Dante e Montale". Dimensioni 5-6(1961): 65-76.

Contini, Gianf ranco. Varianti e altra linguistica. Torino: Einaudi, 1970.

Corti, Maria. Metodi e fantasmi. Milano: Feltrinelli, 1977.

Ierardo, Domenico. Dante nostro contemporaneo. Abano Terme: Piovan, 1987.

Jacomuzzi, Angelo. "Alcune premesse per uno studio sul tema 'Montale e Dante"'. Zennaro 217-27. Luperini, Romano. L' allegoria del moderno. Roma: Editori Riuniti, 1990.

Montale, Eugenio. Sulla poesia. Ed. Giorgio Zampa. Milano: Mondadori, 1976.

Torino: Einaudi, 1980.

Orelli, Giorgio. "L'upupa' e altro". Strumenti critici 15 (1971): 237-63.

. Accertamenti montaliani. Bologna: II Mulino, 1984.

Petrucciani Mario. “Due paragrafi per Dante e il Novecento". Zennaro 163-202.

Pipa, Arshi. Montale and Dante. Minneapolis: U of Minnesota P, 1968.

Ramat, Silvio. "Il Novecento e una traccia dantesca". Forum Italicum 4.3 (1970): 311-30.

Zennaro, Silvio, ed. Dante nella letteratura italiana del Novecento. Atti del Convegno di Studi Casa

di Dante-Roma 6-7 maggio 1977. Roma: Bonacci, 1979. 